\title{
BMJ Open Decision aids for female BRCA mutation carriers: a scoping review protocol
}

\author{
Sarah A McGarrigle (D) , ${ }^{1}$ Geraldine Prizeman, ${ }^{2}$ Carol Spillane, ${ }^{3}$ Niamh Byrne, ${ }^{3}$ \\ Amanda Drury (D) , ${ }^{4,5}$ David Mockler, ${ }^{6}$ Elizabeth M Connolly, ${ }^{3,7}$ Anne-Marie Brady, ${ }^{2}$ \\ Yvonne P Hanhauser ${ }^{5}$
}

To cite: McGarrigle SA, Prizeman G, Spillane C, et al. Decision aids for female BRCA mutation carriers: a scoping review protocol. BMJ Open 2021;11:e045075. doi:10.1136/ bmjopen-2020-045075

- Prepublication history and additional online supplemental material for this paper are available online. To view these files, please visit the journal online (http://dx.doi.org/10. 1136/bmjopen-2020-045075).

A-MB and YPH contributed equally.

Received 22 September 2020 Accepted 15 April 2021

Check for updates

(C) Author(s) (or their employer(s)) 2021. Re-use permitted under CC BY-NC. No commercial re-use. See rights and permissions. Published by BMJ.

For numbered affiliations see end of article.

Correspondence to

Yvonne P Hanhauser;

YHanhauser@stjames.ie

\section{ABSTRACT}

Introduction Women who inherit a pathogenic mutation in Breast Cancer Susceptibility Genes 1 or 2 (BRCA1 or BRCA2) are at substantially higher risk of developing breast and ovarian cancer than the average woman. Several cancer risk management strategies exist to address this increased risk. Decisions about which risk management strategies to choose are complex, personal and multifactorial for these women. This scoping review will map evidence relevant to cancer risk management decision making in BRCA mutation carriers without a personal history of cancer. The objective is to identify and describe the features of patient decision aids that have been developed for BRCA mutation carriers. This information may be beneficial for designing new decision aids or adapting existing decision aids to support decision making in this population.

Methods and analysis This scoping review will be conducted according to the Joanna Briggs Institute's scoping review methodological framework. The Preferred Reporting Items for Systematic Reviews and MetaAnalyses extension for Scoping Reviews checklist will be used for guidance. Studies on decision aids for women with a BRCA mutation who are unaffected by breast or ovarian cancer will be considered for inclusion. Five electronic databases will be searched (MEDLINE, EMBASE, Cochrane Library, CINAHL, Web of Science) with no restrictions applied for language or publication date. Studies for inclusion will be selected independently by two review authors. Data will be extracted using a predefined data extraction form. Findings will be presented in tabular form. A narrative description of the evidence will complement the tabulated results.

Ethics and dissemination Ethical approval for conducting this scoping review is not required as this study will involve secondary analysis of existing literature. Findings will be published in a peer-reviewed journal and presented at relevant conferences.

\section{INTRODUCTION}

\section{Background}

BRCA1 and BRCA2 are tumour suppressor genes that play an important role in the repair of DNA damage. Women who inherit a pathogenic mutation in the BRCA1 or BRCA2 genes are at substantially higher risk of developing

\section{Strengths and limitations of this study}

- This study will provide a comprehensive mapping of literature relevant to cancer risk management decision-making aids in BRCA mutation carriers without a personal history of cancer.

- This scoping review will be conducted according to the Joanna Briggs Institute's scoping review methodological framework and will be guided by the Preferred Reporting Items for Systematic Reviews and Meta-Analyses extension for Scoping Reviews checklist.

- The search strategy will include five databases with no restrictions applied for language or publication date.

- Relevant evidence may exist in the grey literature, but our review is limited to the published literature.

breast and ovarian cancer over their lifetime than the average woman. Estimates for lifetime breast cancer risk vary between studies and differ according to mutation location and family history, but have been reported to be in the region of $45 \%-85 \%$ for female BRCA1 mutation carriers and 27\%-84\% for female BRCA2 carriers to age 70 overall. ${ }^{1-13}$ Furthermore, some studies have reported that BRCA mutation carriers born in recent decades have a substantially higher risk of developing breast cancer than those in earlier birth cohorts. $^{714-16}$

Cumulative ovarian cancer risk to age 80 was estimated to be $44 \%$ for BRCA1 mutation carriers and $17 \%$ for BRCA2 mutations carriers in a study using data from a prospective cohort. ${ }^{1}$ This represents a significant risk compared with a population average of $\leq$ $2 \% .{ }^{17}$

Following a positive genetic test, women diagnosed as BRCA gene mutation carriers may be followed up in high-risk programmes for monitoring and management. Management strategies in this setting are aimed at 
early detection and/or prevention of the disease. Early detection strategies aim to diagnose breast cancer at an early stage to improve clinical outcomes; these include radiologic surveillance at regular intervals by mammography and MRI. Radiological screening techniques have not been proven to be effective in detecting ovarian cancer at an early stage. Prevention strategies aim to reduce a woman's risk of developing breast or ovarian cancer by means of prophylactic surgery (including risk-reducing bilateral mastectomy and/or bilateral salpingo-oophorectomy) or risk-reducing medication (chemoprevention) with drugs such as tamoxifen, anastrozole or raloxifene to reduce breast cancer risk. ${ }^{18}$

For BRCA mutation carriers, decisions about which risk management strategies to choose are complex, personal and multifactorial. Each option has associated risks and anticipated outcomes, which women need to understand to make an informed decision regarding which interventions to choose. Decision Aids in various formats have been developed internationally to support decision-making for BRCA mutation carriers. ${ }^{19-23}$ Such tools require sophisticated design to effectively support decision-making, communicate risk and clarify patients' values and preferences. ${ }^{24}$ Decision aids for BRCA mutation carriers have not yet been widely incorporated into routine clinical practice.

\section{Rationale}

In order to better understand the features of existing decision aids for this population and to reveal which of these decision aids may be appropriate for various populations of BRCA mutation carriers a scoping review of existing decision aids designed to support decision making around risk management for female BRCA mutation carriers will be conducted.

This enquiry is guided by one key question, namely, what are the features of patient decision aids that have been developed to support risk management decisionmaking for female BRCA mutation carriers.

This scoping review will map evidence relevant to cancer risk management decision making supports for female BRCA mutation carriers without a personal history of cancer. This information may be beneficial for designing new decision aids or adapting existing decision aids to support decision-making in terms of cancer risk management for female BRCA mutation carriers.

A preliminary search of the literature (Medline and Embase conducted in July 2020) revealed that a number of decision aids have been developed for this population; therefore, ample evidence sources exist on which to base this review.

The preliminary search did not reveal any existing scoping reviews of patient decision aids developed to support risk management decision making in in BRCA mutation carriers. One quantitative systematic review of effectiveness of decision aids for BRCA mutation carriers that have been tested in randomised control trials or pretest and post-test studies was identified. ${ }^{25}$ The proposed review will differ from this review in that it will summarise the features of all published decision aids for cancer unaffected female BRCA mutation carriers irrespective of whether their effectiveness has been tested in randomised control trials or pre-test and post-test studies.

This scoping review will identify all patient decision aids developed to support decision-making in female BRCA mutation carriers who have not had a cancer diagnosis and will examine the range of features contained in these decision aids. Effectiveness of these decision aids on improving the decision-making process will also be appraised where applicable.

A scoping review can be used to identify, map and discuss certain characteristics in papers or studies. ${ }^{26}$ The aim of this review is to summarise the key characteristics (content and features) of patient decision aids for female BRCA mutation carriers. A scoping review approach can provide a broad overview of the landscape of the literature and is, therefore, the most appropriate design for this evidence synthesis. ${ }^{27}$

\section{Review question}

The question that this scoping review aims to answer is:

What are the features of patient decision aids that have been developed to support risk management decisionmaking in female BRCA mutation carriers.

\section{Objectives}

The objectives of this scoping review are:

(1) to identify and describe the features of patient decision aids that have been developed for female BRCA mutation carriers to support decision-making in terms of choosing which cancer risk management options to opt for.

\section{METHODS AND ANALYSIS}

This scoping review will be conducted according to the Joanna Briggs Institute's scoping review methodological framework. ${ }^{26}$ In addition, the Preferred Reporting Items for Systematic Reviews and Meta-Analyses extension for Scoping Reviews (PRISMA-ScR) checklist will be used for guidance. $^{28}$

\section{Inclusion criteria}

\section{Types of participants}

This review will consider studies on decision aids for cancer risk management in women with a pathogenic BRCA1 or BRCA2 mutation who are unaffected by breast or ovarian cancer.

\section{Concept}

The concept of interest for this scoping review is patient decision aids developed for female BRCA mutation carriers to support their decision-making around cancer risk management options.

\section{Context}

The context of this review is decision-making supports for female BRCA mutation carriers without a personal history 
of breast or ovarian cancer. Sources of evidence on patient decision aids for BRCA mutation carriers pertaining to any contextual setting are eligible for inclusion.

\section{Types of evidence sources}

This review will consider studies that describe the development and/or testing of a patient decision aid for female BRCA mutation carriers to support decision making in terms of choosing which cancer risk management options to opt for. Systematic reviews of the above-mentioned evidence sources will also be considered. This review will not include case reports, non-systematic reviews, protocols, letters, posters or conference abstracts. Studies that describe patient decision aids aimed solely at BRCA mutation carriers with a personal history of breast or ovarian cancer will be excluded also.

\section{Search strategy}

A three-step search strategy will be used. First, an initial limited search of the databases MEDLINE (Ovid) and EMBASE was conducted. This initial search was followed by an analysis of the text words contained in the title and abstract of retrieved papers, and of the index terms used to describe the articles. A second search using identified keywords and index terms (online supplemental appendix 1) will then be undertaken across all included databases (MEDLINE, EMBASE, Cochrane Library, CINAHL, Web of Science) (online supplemental appendix 1). Databases will be searched from inception to present. No restrictions will be applied for language or publication date. The National Institute for Health and Care Excellence guidelines ${ }^{18}$ will also be searched for relevant evidence. Thirdly, the reference list of reports and articles selected for inclusion in the review will be searched for additional sources.

\section{Source of evidence selection}

Search results will be uploaded to EndNote X8 (Clarivate Analytics, Pennsylvania, USA) and duplicate records will be removed. Retrieved studies will initially be screened for inclusion by title and abstract by two review authors independently using the web based Covidence screening tool (Veritas Health Innovation, Melbourne, Australia). Disagreements will be resolved by discussion. Full text papers and reports will be retrieved for potentially relevant studies. For these studies, Covidence software will once again be used to assess and document studies for inclusion and exclusion according to the inclusion criteria. Studies for inclusion will be selected independently by two review authors. Disagreements will be resolved by discussion. In cases of no consensus, final resolution will be achieved by involving a third review author as arbiter. Study selection will be documented in a PRISMA-ScR flow chart. Reasons for exclusion of articles will be included in the scoping review report.

\section{Data extraction}

Data will be extracted from included articles and other evidence sources using a data extraction form developed by the reviewers (online supplemental appendix 2). The design of this instrument is based on the Joanna Briggs Institute instrument for extracting details of the studies characteristics and results. Data extraction will be performed independently by two reviewers. Disagreements that arise between the reviewers will be resolved through discussion, or by involving a third review author as arbiter. Key items to extract include: author, year, country, type of document, objective, features/components of patient decision aids for BRCA mutation carriers, format of patient decision aids, patient and public involvement in decision aid development, efficacy of patient decision aids (where applicable). Data extracted will include specific details about the population, sample size (where applicable), study methods and key findings relevant to the scoping review objectives. The data extraction tool will be modified and revised as required during the process of data extraction. Modifications will be detailed in the full scoping review report. In cases where pertinent information is not reported efforts will be made to contact study authors to request this information.

\section{Analysis of the evidence \\ Data presentation}

Extracted data will be presented in tabular form in a manner that aligns with the objective of this scoping review. A narrative description of the evidence will complement the tabulated results. In addition, a description of how the results answer the objectives and questions of this review will be provided. The review findings and their implications for research and practice in the field of decision-making for female BRCA mutation carriers will be discussed.

\section{PATIENT AND PUBLIC INVOLVEMENT}

Patients and public were not formally involved in the development of this scoping review protocol; however, the research questions were informed by the author team's extensive clinical experience working with BRCA mutation carriers.

\section{ETHICS AND DISSEMINATION}

Ethical approval for the conduct of this scoping review is not required as this study will involve secondary analysis of the existing literature. Findings will be published in a peerreviewed journal and presented at relevant conferences. Findings will be disseminated further through social media platforms.

\section{DISCUSSION}

This scoping review will fill an important gap in the literature, as no scoping reviews of evidence relevant to cancer risk management decision-making supports for BRCA mutation carriers currently exist that map the features of patient decision aids developed for these women.

As a scoping review, results from this study will provide a descriptive overview of available evidence. An additional limitation of this review is that we will limit our search to 
the published literature, which could potentially result in relevant evidence in the grey literature being overlooked.

Overall, evidence collated through this scoping review may be beneficial for designing new patient decision aids or adapting existing decision aids to support decision-making in terms of cancer risk management for female BRCA mutation carriers.

\section{Author affiliations}

${ }^{1}$ Faculty of Health Sciences, Trinity College Dublin, Dublin, Ireland

${ }^{2}$ Trinity Centre for Practice and Healthcare Innovation, School of Nursing \& Midwifery, Trinity College Dublin, Dublin, Ireland

${ }^{3}$ Breast Care Department, St. James's Hospital, Dublin, Ireland

${ }^{4}$ School of Nursing, Midwifery and Health Systems, University College Dublin, Dublin, Ireland

${ }^{5}$ School of Nursing and Midwifery, Trinity College Dublin, Dublin, Ireland

${ }^{6}$ John Stearne Library, Trinity College Dublin, Dublin, Ireland

${ }^{7}$ Department of Surgery, Trinity College Dublin, Dublin, Ireland

\section{Twitter Sarah A McGarrigle @drsmcgarrigle and Amanda Drury @druryal}

Contributors YPH and A-MB led the conceptualisation, design and development of this study. GP conceptualised the scoping review approach. YPH, A-MB, SAM, GP, $C S, N B$ and $A D$ were involved in developing the review questions. SAM drafted the protocol. DM developed the search strategy with input from SAM. EMC provided feedback on the study design from a clinical perspective. All authors reviewed and approved the final version of the manuscript.

Funding This work is supported by an Irish Cancer Society Research Grant, CNRA19HAN.

\section{Competing interests None declared.}

Patient consent for publication Not required.

Provenance and peer review Not commissioned; externally peer reviewed.

Supplemental material This content has been supplied by the author(s). It has not been vetted by BMJ Publishing Group Limited (BMJ) and may not have been peer-reviewed. Any opinions or recommendations discussed are solely those of the author(s) and are not endorsed by BMJ. BMJ disclaims all liability and responsibility arising from any reliance placed on the content. Where the content includes any translated material, BMJ does not warrant the accuracy and reliability of the translations (including but not limited to local regulations, clinical guidelines, terminology, drug names and drug dosages), and is not responsible for any error and/or omissions arising from translation and adaptation or otherwise.

Open access This is an open access article distributed in accordance with the Creative Commons Attribution Non Commercial (CC BY-NC 4.0) license, which permits others to distribute, remix, adapt, build upon this work non-commercially, and license their derivative works on different terms, provided the original work is properly cited, appropriate credit is given, any changes made indicated, and the use is non-commercial. See: http://creativecommons.org/licenses/by-nc/4.0/.

\section{ORCID iDs}

Sarah A McGarrigle http://orcid.org/0000-0003-4404-6564

Amanda Drury http://orcid.org/0000-0002-1720-5673

\section{REFERENCES}

1 Kuchenbaecker KB, Hopper JL, Barnes DR, et al. Risks of breast, ovarian, and contralateral breast cancer for BRCA1 and BRCA2 mutation carriers. JAMA 2017;317:2402-16.

2 Chen S, Iversen ES, Friebel T, et al. Characterization of BRCA1 and BRCA2 mutations in a large United States sample. J Clin Oncol 2006;24:863-71.

3 Milne RL, Osorio A, Cajal TRY, et al. The average cumulative risks of breast and ovarian cancer for carriers of mutations in BRCA1 and BRCA2 attending genetic counseling units in Spain. Clin Cancer Res 2008;14:2861-9.

4 Chen J, Bae E, Zhang L, et al. Penetrance of Breast and Ovarian Cancer in Women Who Carry a BRCA1/2 Mutation and Do Not Use
Risk-Reducing Salpingo-Oophorectomy: An Updated Meta-Analysis. JNCl Cancer Spectr 2020;4:pkaa029.

5 Antoniou A, Pharoah PDP, Narod S, et al. Average risks of breast and ovarian cancer associated with BRCA1 or BRCA2 mutations detected in case series unselected for family history: a combined analysis of 22 studies. Am J Hum Genet 2003;72:1117-30.

6 Antoniou AC, Durocher F, Smith P, et al. Brca1 and BRCA2 mutation predictions using the BOADICEA and BRCAPRO models and penetrance estimation in high-risk French-Canadian families. Breast Cancer Res 2006;8:R3.

7 Evans DG, Shenton A, Woodward E, et al. Penetrance estimates for BRCA1 and BRCA2 based on genetic testing in a clinical cancer genetics service setting: risks of breast/ovarian cancer quoted should reflect the cancer burden in the family. BMC Cancer 2008;8:155.

8 Mavaddat N, Peock S, Frost D, et al. Cancer risks for BRCA1 and BRCA2 mutation carriers: results from prospective analysis of embrace. J Nat/ Cancer Inst 2013;105:812-22.

9 Chen S, Parmigiani G. Meta-Analysis of BRCA1 and BRCA2 penetrance. J Clin Oncol 2007;25:1329-33.

10 Brohet RM, Velthuizen ME, Hogervorst FBL, et al. Breast and ovarian cancer risks in a large series of clinically ascertained families with a high proportion of BRCA1 and BRCA2 Dutch founder mutations. $J$ Med Genet 2014;51:98-107.

11 Easton DF, Ford D, Bishop DT. Breast and ovarian cancer incidence in BRCA1-mutation carriers. breast cancer linkage Consortium. Am J Hum Genet 1995;56:265-71.

12 Gabai-Kapara E, Lahad A, Kaufman B, et al. Population-Based screening for breast and ovarian cancer risk due to BRCA1 and BRCA2. Proc Natl Acad Sci U S A 2014;111:14205-10.

13 Ford D, Easton DF, Stratton M, et al. Genetic heterogeneity and penetrance analysis of the BRCA1 and BRCA2 genes in breast cancer families. the breast cancer linkage Consortium. Am J Hum Genet 1998;62:676-89.

14 Tryggvadottir L, Sigvaldason H, Olafsdottir GH, et al. PopulationBased study of changing breast cancer risk in Icelandic BRCA2 mutation carriers, 1920-2000. J Natl Cancer Inst 2006;98:116-22.

15 King M-C, Marks JH, Mandell JB, et al. Breast and ovarian cancer risks due to inherited mutations in BRCA1 and BRCA2. Science 2003;302:643-6.

16 Berrino J, Berrino F, Francisci S, et al. Estimate of the penetrance of BRCA mutation and the COS software for the assessment of BRCA mutation probability. Fam Cancer 2015;14:117-28.

17 Nash Z, Menon U. Ovarian cancer screening: current status and future directions. Best Pract Res Clin Obstet Gynaecol 2020;65:32-45

18 NICE. National Institute for Health and Care Excellence (NICE) (2013). Familial breast cancer: classification, care and managing breast cancer and related risks in people with a family history of breast cancer. Clinical guideline [CG164], 2013.

19 Kaufman EM, Peshkin BN, Lawrence WF, et al. Development of an interactive decision aid for female BRCA1/BRCA2 carriers. J Genet Couns 2003:12:109-29.

20 Culver JO, MacDonald DJ, Thornton AA, et al. Development and evaluation of a decision aid for BRCA carriers with breast cancer. $J$ Genet Couns 2011;20:294-307.

21 Kurian AW, Munoz DF, Rust P, et al. Online tool to guide decisions for BRCA1/2 mutation carriers. J Clin Oncol 2012;30:497-506.

22 Katapodi MC, Jung M, Schafenacker AM, et al. Development of a web-based family intervention for BRCA carriers and their biological relatives: acceptability, feasibility, and usability study. JMIR Cancer 2018;4:e7.

23 Jabaley T, Underhill-Blazey ML, Berry DL. Development and testing of a decision aid for unaffected women with a BRCA1 or BRCA2 mutation. J Cancer Educ 2020;35:339-44.

24 Silverman TB, Kuperman GJ, Vanegas A, et al. An Applied Framework in Support of Shared Decision Making about $B R C A$ Genetic Testing. AMIA Annu Symp Proc 2018;2018:961-9.

25 Krassuski L, Vennedey V, Stock S, et al. Effectiveness of decision AIDS for female BRCA1 and BRCA2 mutation carriers: a systematic review. BMC Med Inform Decis Mak 2019;19:154.

26 Peters MDJ, Godfrey CM, Mclnerney P. Chapter 11: Scoping Reviews. In: Aromataris E, Munn Z, eds. Joanna Briggs Institute Reviewer's Manual, JBI, 2020.

27 Munn Z, Peters MDJ, Stern C, et al. Systematic review or scoping review? guidance for authors when choosing between a systematic or scoping review approach. BMC Med Res Methodol 2018;18:143.

28 Tricco AC, Lillie E, Zarin W, et al. PRISMA extension for scoping reviews (PRISMA-ScR): checklist and explanation. Ann Intern Med 2018;169:467-73. 